\title{
Compliance abhängig von Farbe und Form der Tabletten?
}

\author{
Läuft das Patent für ein Originalmedikament aus, kommen
}

i. d. R. entsprechende Generika in den Handel. Mit dem Druck zur Kosteneinsparung im Gesundheitswesen nimmt der Anteil

an Generika immer mehr zu. Generika müssen zwar in

Bioäquivalenzstudien nachweisen, dass ihre Freisetzungs-

kinetik und Bioverfügbarkeit denen der Originalpräparate entsprechen. Aber genügt das? Mit dieser Frage haben sich

A. S. Kesselheim et al. beschäftigt. JAMA Intern Med 2013; 173: 202-208

Veränderungen der Tablettenfarbe erhöhen das Risiko einer Nicht-Einnahme - so die Ergebnisse der Fall-Kontroll-Studie aus den USA, in der Daten von mehr als 60000 Patienten ausgewertet wurden. Als Beispiel wurde die Einnahme von Antiepileptika überprüft, da hier eine exakte Einnahme von besonderer Bedeutung ist. Schon kurze Überschreitungen des Einnahmeintervalls können zu erheblichen medizischen, finanziellen und sozialen Konsequenzen führen.

In die Auswertung aufgenommen wurden 11472 Patienten mit regelmäßiger Einnahme von Antikonvulsiva (wegen einer Epilepsie, aber auch chronischer Schmerzsyndrome, affektiver und anderer psychischer Störungen), die ihr Rezept nicht einlösten, nachdem eine Packung (rein rechnerisch) aufgebraucht war. 50050 Patienten ohne eine solche Unterbrechung dienten als Kontrollgruppe. Die Forscher bestimmten anschließend, ob vor diesem Nicht-Einlösen des Rezepts ein Wechsel des Präparats mit Veränderungen von Form und/oder

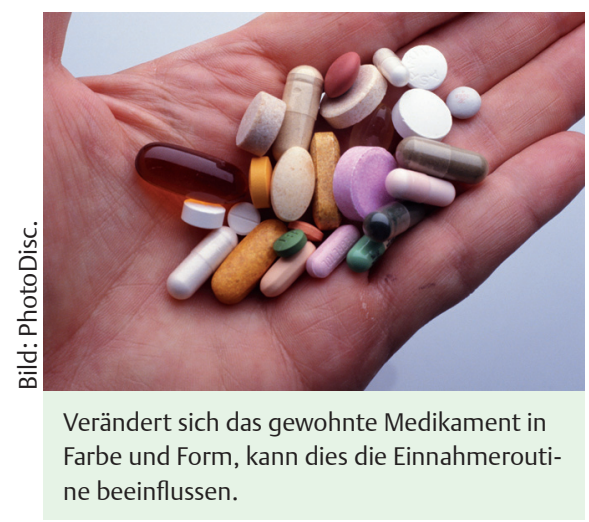

Farbe der Tabletten vorgekommen war und berechneten das Risiko für eine Nicht-Einnahme in Abhängigkeit davon.

Es zeigte sich, dass eine Veränderung der Tablettenfarbe bei 136 Fällen (1,2\%) dem Nicht-Einlösen des Rezepts vorangegangen war. In der Kontrollgruppe fand sich eine derartige Veränderung bei 480 Teilnehmern (0,97\%; adjustierte Odds Ratio [OR] 1,27; 95\%-Konfidenzintervall [KI] 1,04-1,55). Eine Veränderung der Tablettenform war weniger häufig: Sie ging bei 18 Patienten einem Nicht-Einlösen voraus $(0,16 \%)$, gegenüber 54 Kontrollpatienten (0,11\%; OR 1,47; 95\%-KI 0,85-2,54). Auch in der Subgruppe der Patienten mit tatsächlicher Epilepsiediagnose waren die Verhältnisse ähnlich: Die OR für eine Nicht-Einnahme lag bei 1,53 (95\%-KI 1,072,18 ) nach veränderter Tablettenfarbe.

\section{Fazit}

Die Non-Compliance bei Patienten, die Antikonvulsiva einnehmen, könnte durch eine neue Tablettenfarbe steigen, so die Autoren. Einschränkend fügen sie hinzu, dass die Parameter, mit denen die Compliance beurteilt wurde, mit Fehlern behaftet sein könnten - möglicherweise wurde das Einnahmeschema vom Arzt geändert. Ebenso gewährleistet eine Rezepteinlösung nicht die tatsächliche Einnahme der Tabletten. Dennoch könnte ein Überdenken der Zulassungsvoraussetzungen für Generika im Hinblick auf das Aussehen sinnvoll sein.

Dr. Elke Ruchalla, Trossingen
Allergologie

\section{Neue Risikogene identifiziert}

Ein internationales Forscherteam hat in einer Analyse mehrerer Genom-Studien 10 Gene gefunden, die für die Entstehung einer allergischen Sensibilisierung verantwortlich sind. Ihre Ergebnisse wurden Anfang Juli in der Fachzeitschrift „Nature Genetics“ veröffentlicht. In den Untersuchungen, sog. genomweiten Assoziationsstudien (GWAs), verglichen die Wissenschaftler das genetische Profil der Studienteilnehmer mit dem Auftreten allergietypischer Antikörper. Sie stellten dabei fest, dass 10 Genorte an insgesamt 25\% aller allergischen Sensibilisierungen beteiligt sind.

Die Wissenschaftler um K. Bønnelykke aus Kopenhagen analysierten hierfür die Daten von über 10000 Personen mit einer allergischen Sensibilisierung und ca. 20000 Kontrollpersonen. Darunter waren auch Teilnehmer der deutschen Neugeborenen-Kohorten GINIplus und LISAplus, deren Auswertung durch Marie Standl und Dr. Joachim Heinrich vom Institut für Epidemiologie I (EPI I) am Helmholtz Zentrum München erfolgte. Diese umfassende Genanalyse lässt eine Bewertung der identifizierten Gene als Risikofaktoren für allergische Erkrankungen zu. Zudem zeigten alle diese Gene einen $\mathrm{Zu}$ sammenhang mit dem Auftreten allergischer Symptome, wie Heuschnupfen oder Asthma bronchiale.

Ursächlich für das allergische Geschehen ist eine komplexe Gen-Umwelt-Interaktion. „Die Ergebnisse zeigen, dass allergischen Erkrankungen eine enorme genetische Vielfalt zugrunde liegt. Weiterhin spielen Lebensstilfaktoren und Umweltbelastungen in der künftigen Allergieforschung und -behandlung eine wichtige Rolle“, erklärt Heinrich. Die Wissenschaftler wollen nun anhand der genetischen Informationen die beteiligten molekularen Mechanismen weiter untersuchen, um neue Einblicke in die Entstehung von Allergien und anderen Immunerkrankungen zu erlangen.

Nach einer Mitteilung des Helmholtz Zentrums München 